Article

\title{
Oscillatory Properties of Odd-Order Delay Differential Equations with Distribution Deviating Arguments
}

\author{
Ali Muhib ${ }^{1,2, *} \mathbb{D}$, Thabet Abdeljawad ${ }^{3,4,5, *} \mathbb{C}$, Osama Moaaz ${ }^{1} \mathbb{D}$ and Elmetwally M. Elabbasy ${ }^{1}$ \\ 1 Department of Mathematics, Faculty of Science, Mansoura University, Mansoura 35516, Egypt; \\ o_moaaz@mans.edu.eg (O.M.); emelabbasy@mans.edu.eg (E.M.E.) \\ 2 Department of Mathematics, Faculty of Education-Al-Nadirah, Ibb University, Ibb P.O. Box 70270, Yemen \\ 3 Department of Mathematics and General Sciences, Prince Sultan University, Riyadh 11586, Saudi Arabia \\ 4 Department of Medical Research, China Medical University, Taichung 40402, Taiwan \\ 5 Department of Computer Science and Information Engineering, Asia University, Taichung 40402, Taiwan \\ * Correspondence: muhib39@yahoo.com or muhib39@students.mans.edu.eg (A.M.); \\ tabdeljawad@psu.edu.sa (T.A.)
}

Received: 28 June 2020; Accepted: 26 August 2020; Published: 27 August 2020

check for updates

\begin{abstract}
Throughout this work, new criteria for the asymptotic behavior and oscillation of a class of odd-order delay differential equations with distributed deviating arguments are established. Our method is essentially based on establishing sharper estimates for positive solutions of the studied equation, using an iterative technique. Moreover, the iterative technique allows us to test the oscillation, even when the related results fail to apply. By establishing new comparison theorems that compare the nth-order equations with one or a couple of first-order delay differential equations, we obtain new conditions for oscillation of all solutions of the studied equation. To show the importance of our results, we provide two examples.
\end{abstract}

Keywords: positive solutions; delay differential equations; odd-order

\section{Introduction}

In this work, we study the asymptotic and oscillatory behavior of odd-order delay differential equations with distributed deviating arguments of the form

$$
\left(r(\xi)\left(x^{(n-1)}(\xi)\right)^{\alpha}\right)^{\prime}+\int_{a}^{b} \rho(\xi, s) f(x(\psi(\xi, s))) \mathrm{d} s=0,
$$

where $n \in \mathbb{Z}^{+}$is an odd. Moreover, we assume the following conditions:

(i) $\quad r \in C^{1}\left(\left[\xi_{0}, \infty\right),(0, \infty)\right), r^{\prime}(\xi) \geq 0, \alpha$ is a ratio of odd positive integers and

$$
\mu_{0,0}\left(\xi, \xi_{0}\right):=\int_{\xi_{0}}^{\xi} r^{-1 / \alpha}(s) \mathrm{d} s \rightarrow \infty \text { as } \xi \rightarrow \infty ;
$$

(ii) $\quad \psi, \rho \in C\left(\left[\xi_{0}, \infty\right) \times[a, b], \mathbb{R}\right), \rho(\xi, s) \geq 0, \psi(\xi, s) \leq \xi$ for $\xi \geq \xi_{0}$ and $s \in[a, b]$, $\psi$ has nonnegative partial derivatives and $\lim _{\xi \rightarrow \infty} \psi(\xi, s)=\infty$;

(iii) $f \in C(\mathbb{R}, \mathbb{R}), f(\xi, x) \geq \varrho x^{\alpha}$ for $x \neq 0$ and $\varrho$ is a positive constant.

The function $x$ is said to be a solution of Equation (1) if there exists a $\xi_{x} \geq \xi_{0}$ such that $x \in$ $C^{n-1}\left(\left[\xi_{x}, \infty\right), \mathbb{R}\right), r(\xi)\left(x^{(n-1)}\right)^{\alpha} \in C^{1}\left(\left[\xi_{x}, \infty\right), \mathbb{R}\right)$ and satisfies Equation $(1)$, for all $\xi \in\left[\xi_{x}, \infty\right)$. 
We limit our discussion to those solutions $x$ of Equation (1) which satisfy $\sup \left\{|x(\xi)|: \xi_{1} \leq \xi_{0}\right\}>0$ for every $\xi_{1} \in\left[\xi_{x}, \infty\right)$. Such a solution $x$ is said to be non-oscillatory if $x$ is positive or negative, ultimately; otherwise, $x$ is said to be oscillatory. If every solutions of Equation (1) is oscillatory, then Equation (1) is called oscillatory.

Oscillatory behavioral nature of solutions of various classes of neutral and delay differential equations is of great interest, and often encountered in applied problems in natural sciences, technology, and engineering, see [1,2]. Recently it has been noticed the rising interest of many researchers and papers in studying the qualitative properties of different classes of linear and non-linear differential equations, see [1-14].

To the best of the authors' knowledge, the study and examination of the oscillatory behavior of odd-order differential equations received less attention than even-order, see [15-25].

Vidhyaa et al. [26] studied the oscillation of all solutions of the third-order neutral differential equation

$$
\left(\psi(\xi)\left(\left(h(\xi) z^{\prime}(\xi)\right)^{\prime}\right)^{\alpha}\right)^{\prime}+f(\xi) y^{\alpha}(\xi)=0, \xi \geq \xi_{0}
$$

where $z(\xi)=y(\xi)+p(\xi) y(\sigma(\xi))$.

$\mathrm{Li}$ and Rogovchenko [27] investigated asymptotic behavior of solutions to an odd-order delay differential equation

$$
\left(r(\xi)\left(x^{(n-1)}(\xi)\right)^{\alpha}\right)^{\prime}+p(\xi)\left(x^{(n-1)}(\xi)\right)^{\alpha}+\rho(\xi) x^{\alpha}(\tau(\xi))=0,
$$

where $\xi \geq \xi_{0}>0$ and $n \geq 3$ is an odd natural number.

Baculikova and Dzurina [18] adopted the Riccati transformation for examining the asymptotic and oscillation behavior of the solutions of advanced differential equations of higher order

$$
\left(r(\xi)\left(x^{(n-1)}(\xi)\right)^{\alpha}\right)^{\prime}+\rho(\xi) x^{\alpha}(\tau(\xi))=0,
$$

where $\tau(\xi)>\xi$ and $n$ is odd.

Based on the use of iterative technique, we first establish a more sharp estimate of the increasing/decreasing positive solutions of Equation (1). By following the comparison approach with first order delay equations, we attain oscillation of all solutions of Equation (1) under easy application conditions.

In the following, we present some useful lemmas that will be used throughout the results.

Lemma 1. (Lemma 2.2.3 in [3]) If $F \in C^{n}\left(\left[\xi_{0}, \infty\right),(0, \infty)\right), F^{(n-1)}(\xi) F^{(n)}(\xi) \leq 0$ for $\xi \geq \xi_{F} \geq \xi_{0}$ and $\lim _{\tilde{\xi} \rightarrow \infty} F(\xi) \neq 0$, then there exists a $\xi_{\delta} \in\left[\xi_{F}, \infty\right)$ such that

$$
F(\xi) \geq \frac{\delta}{(n-1) !} \xi^{n-1}\left|F^{(n-1)}(\xi)\right|
$$

for every $\delta \in(0,1)$ and $\xi \in\left[\xi_{\delta}, \infty\right)$.

Lemma 2. (Lemma 2 in [18]) If $x$ is a positive solution of Equation (1), then all derivatives $x^{(k)}(\xi), 1 \leq k \leq$ $n-1$, are of constant signs, $r(\xi)\left(x^{(n-1)}(\xi)\right)^{\alpha}$ is non-increasing, and $x$ satisfies either

$$
x^{\prime}(\xi)>0, x^{\prime \prime}(\xi)>0, x^{(n-1)}(\xi)>0, x^{(n)}(\xi)<0
$$

or

$$
(-1)^{m} x^{(m)}>0, m=1,2, \ldots, n .
$$


Next, we provide the following notations to help us display the results easily:

$$
\rho_{k}(u):=\varrho \int_{a}^{b} \rho(u, s) \eta_{k}^{\alpha}(\psi(u, s)) \mathrm{d} s,
$$

moreover, we denoted the set of all positive solutions of Equation (1) with property Equation (2) or Equation (3) by $X_{I}^{+}$or $X_{D}^{+}$, respectively.

Remark 1. All functional inequalities and properties including increasing, decreasing, positive, etc. are assumed to hold eventually, that is, they are satisfied for all $\xi$ large enough.

\section{Nonexistence of Increasing Positive Solutions}

In this section, we obtain nonexistence criteria for increasing positive solutions of odd-order delay differential Equation (1).

Lemma 3. Assume that $x \in X_{I}^{+}$. Then,

$$
x(\psi(\xi, s)) \geq \eta_{k}(\psi(\xi, s)) x^{(n-1)}(\psi(\xi, s)),
$$

where

$$
\eta_{0}(\xi):=\frac{\delta_{0}}{(n-1) !} \xi^{n-1}
$$

and

$$
\eta_{k+1}(\xi):=\left(\frac{\delta_{k} r^{1 / \alpha}(\xi)}{(n-2) !}\right) \int_{\xi_{1}}^{\xi} v^{n-2}\left(\frac{1}{r(v)} \exp \left(\int_{v}^{\tau} \frac{\rho_{k}(u)}{r(u)} \mathrm{d} u\right)\right)^{1 / \alpha} \mathrm{d} v
$$

for all $\delta_{k} \in(0,1)$ and $k=0,1, \ldots$

Proof. Assume that $x \in X_{I}^{+}$. Then, there exists a $\xi_{1} \geq \xi_{0}$ such that $x(\xi)>0$ and $x(\psi(\xi, s))>0$ for all $\xi \geq \xi_{1}$. Now, we will use the induction to prove Equation (4). For $k=0$, using Lemma 1 , we obtain

$$
x(\psi(\xi, s)) \geq \frac{\delta_{0}}{(n-1) !} \psi^{n-1}(\xi, s) x^{(n-1)}(\psi(\xi, s)) \geq \eta_{0}(\psi(\xi, s)) x^{(n-1)}(\psi(\xi, s)) .
$$

Next, we assume that $x(\psi(\xi, s)) \geq \eta_{k}(\psi(\xi, s)) x^{(n-1)}(\psi(\xi, s))$, for $k>1$. Then

$$
x^{\alpha}(\psi(\xi, s)) \geq \eta_{k}^{\alpha}(\psi(\xi, s))\left(x^{(n-1)}(\psi(\xi, s))\right)^{\alpha}
$$

or equivalently,

$$
f(x(\psi(\xi, s))) \geq \varrho \eta_{k}^{\alpha}(\psi(\xi, s))\left(x^{(n-1)}(\psi(\xi, s))\right)^{\alpha} .
$$

Combining Equations (1) and (5), we get

$$
\left(r(\xi)\left(x^{(n-1)}(\xi)\right)^{\alpha}\right)^{\prime} \leq-\varrho \int_{a}^{b} \rho(\xi, s) \eta_{k}^{\alpha}(\psi(\xi, s))\left(x^{(n-1)}(\psi(\xi, s))\right)^{\alpha} \mathrm{d} s .
$$

Since $x^{(n)}<0$ and $\psi(\xi, a)<\xi$, we have

$$
\left(r(\xi)\left(x^{(n-1)}(\xi)\right)^{\alpha}\right)^{\prime} \leq-\varrho\left(x^{(n-1)}(\xi)\right)^{\alpha} \int_{a}^{b} \rho(\xi, s) \eta_{k}^{\alpha}(\psi(\xi, s)) \mathrm{d} s .
$$


If we set $w:=r(\xi)\left(x^{(n-1)}(\xi)\right)^{\alpha}$, then (6) reduces to

$$
w^{\prime}(\xi) \leq-\frac{\varrho}{r(\xi)} w(\xi) \int_{a}^{b} \rho(\xi, s) \eta_{k}^{\alpha}(\psi(\xi, s)) \mathrm{d} s .
$$

By using Grönwall inequality, we obtain

$$
w(v) \geq w(\xi) \exp \left(\int_{v}^{\xi} \frac{\rho_{k}}{r(u)} \mathrm{d} u\right)
$$

and hence

$$
x^{(n-1)}(v) \geq\left(r^{1 / \alpha}(\xi) x^{(n-1)}(\xi)\right)\left(\frac{1}{r(v)} \exp \left(\int_{v}^{\xi} \frac{\rho_{k}}{r(u)} \mathrm{d} u\right)\right)^{1 / \alpha} .
$$

Using Lemma 1 with $F:=x^{\prime}>0$, we conclude that

$$
x^{\prime}(\xi) \geq \frac{\delta_{k} \xi^{n-2}}{(n-2) !} x^{(n-1)}(\xi), \text { for all } \delta_{k} \in(0,1) .
$$

Integrating (8) from $\xi_{1}$ to $\xi$, and using (7), we find

$$
\begin{aligned}
x(\xi) & \geq \frac{\delta_{k}}{(n-2) !} \int_{\xi_{1}}^{\xi} v^{n-2} x^{(n-1)}(v) \mathrm{d} v \\
& \geq\left(x^{(n-1)}(\xi) \frac{\delta_{k} r^{1 / \alpha}(\xi)}{(n-2) !}\right) \int_{\xi_{1}}^{\xi} v^{n-2}\left(\frac{1}{r(v)} \exp \left(\int_{v}^{\xi} \frac{\rho_{k}}{r(u)} \mathrm{d} u\right)\right)^{1 / \alpha} \mathrm{d} v \\
& \geq \eta_{k+1}(\xi) x^{(n-1)}(\xi),
\end{aligned}
$$

or equivalently,

$$
x(\psi(\xi, s)) \geq \eta_{k+1}(\psi(\xi, s)) x^{(n-1)}(\psi(\xi, s)) .
$$

The proof is complete.

Theorem 1. Assume that $\eta_{k}$ is defined as in Lemma 3. If, for some $\delta_{k} \in(0,1)$ and some $k \in \mathbb{N}$, the delay differential equation

$$
w^{\prime}(\xi)+\frac{\rho_{k}(u)}{r(\psi(\xi, a))} w(\psi(\xi, a))=0
$$

is oscillatory, then $X_{I}^{+}$is empty.

Proof. Assume the contrary that $x \in X_{I}^{+}$. Then, there exists a $\xi_{1} \geq \xi_{0}$ such that $x(\xi)>0$ and $x(\psi(\xi, s))>0$ for all $\xi \geq \xi_{1}$. As in the proof of Lemma 3, we arrive at (6). Since $\psi(\xi, s)$ is nondecreasing with respect to $s$, we get that $\psi(\xi, s) \geq \psi(\xi, a)$ for $s \in(a, b)$. Then, (6) becomes

$$
\left(r(\xi)\left(x^{(n-1)}(\xi)\right)^{\alpha}\right)^{\prime} \leq-\varrho\left(x^{(n-1)}(\psi(\xi, a))\right)^{\alpha} \int_{a}^{b} \rho(\xi, s) \eta_{k}^{\alpha}(\psi(\xi, s)) \mathrm{d} s .
$$

If we set $w:=r\left(x^{(n-1)}\right)^{\alpha}>0$, then (10) becomes

$$
w^{\prime}(\xi)+\frac{\rho_{k}(\xi)}{r(\psi(\xi, a))} w(\psi(\xi, a)) \leq 0 .
$$

Thus, the last inequality has a positive solution. Using Theorem 1 in [10], we see that (9) also has a positive solution, which is a contradiction. The proof is complete. 
Corollary 1. Assume that $\eta_{k}$ is defined as in Lemma 3. If, for some $\delta_{k} \in(0,1)$ and some $k \in \mathbb{N}$,

$$
\liminf _{\tilde{\zeta} \rightarrow \infty} \int_{\psi(\xi, a)}^{\xi} \frac{\rho_{k}(u)}{r(\psi(u, a))} \mathrm{d} u>\frac{1}{\mathrm{e}^{\prime}}
$$

then $X_{I}^{+}$is empty.

Proof. Applying a well-known criterion Theorem 2 in [13] for first-order delay differential Equation (9) to be oscillatory, we obtain immediately the criterion (11).

Example 1. Consider the third-order differential equation

$$
x^{\prime \prime \prime}+\int_{\epsilon}^{1} \frac{\rho_{0}}{\bar{\zeta}^{3}} x(\epsilon \xi) \mathrm{d} \xi=0,
$$

where $\xi \geq 1, \alpha=1, n=3, r=1, \rho(\xi, s)=\rho_{0} / \xi^{3}, \rho_{0}>0, a=\epsilon, b=1, \psi(\xi, s)=\epsilon \xi$ and $\epsilon \in(0,2 / 3)$. Then, we get that

$$
\eta_{0}(\xi):=\frac{\delta_{0}}{2} \epsilon^{2} \xi^{2}
$$

It is easy to verify that condition (11) with $k=0$ reduces to

$$
\varrho \rho_{0} \delta_{0} \epsilon^{2}(1-\epsilon) \ln \frac{1}{\epsilon}>\frac{2}{\mathrm{e}}
$$

From Corollary 1, we see that $X_{I}^{+}$is empty if (13) satisfies.

\section{Nonexistence of Decreasing Positive Solutions}

In this section, we obtain nonexistence criteria for decreasing positive solutions of odd-order delay differential Equation (1).

Lemma 4. Assume that $x \in X_{D}^{+}$. Then,

$$
x(u) \geq r^{1 / \alpha}(v) x^{(n-1)}(v) \mu_{l, n-2}(v, u),
$$

where

$$
\mu_{l, k+1}(v, u):=\int_{u}^{v} \mu_{l, k}(v, s) \mathrm{d} s
$$

and

$$
\mu_{l+1,0}(v, u):=\int_{u}^{v}\left(\frac{1}{r(v)} \exp \left(\int_{v}^{v}\left(\varrho \int_{a}^{b} \rho(u, s) \mu_{l, n-2}^{\alpha}(u, \psi(u, s)) \mathrm{d} s\right) \mathrm{d} u\right)\right)^{1 / \alpha} \mathrm{d} v,
$$

for $k=0,1, \ldots, n-3$, and $l=0,1,2, \ldots$

Proof. Assume that $x \in X_{D}^{+}$. Then, there exists a $\xi_{1} \geq \xi_{0}$ such that $x(\xi)>0$ and $x(\psi(\xi, s))>0$ for all $\xi \geq \xi_{1}$. Now, we use the induction to prove (14). For $l=0$, since $\left(r\left(x^{(n-1)}\right)\right)^{\prime} \leq 0$, we get

$$
\begin{aligned}
-x^{(n-2)}(u) & \geq x^{(n-2)}(v)-x^{(n-2)}(u)=\int_{u}^{v} \frac{1}{r^{1 / \alpha}(s)} r^{1 / \alpha}(s) x^{(n-1)}(s) \mathrm{d} s \\
& \geq r^{1 / \alpha}(v) x^{(n-1)}(v) \mu_{0,0}(v, u) .
\end{aligned}
$$

Integrating (15) from $u$ to $v$, we have that

$$
-x^{(n-3)}(u) \leq x^{(n-3)}(v)-x^{(n-3)}(u)=r^{1 / \alpha}(v) x^{(n-1)}(v) \mu_{0,1}(v, u) .
$$


Integrating (16) $(n-3)$ times from $u$ to $v$, we conclude that

$$
x(u) \geq r^{1 / \alpha}(v) x^{(n-1)}(v) \mu_{0, n-2}(v, u) .
$$

Next, we assume that $x(u) \geq r^{1 / \alpha}(v) x^{(n-1)}(v) \mu_{l, n-2}(v, u)$, for $l>1$. Thus, we obtain

$$
x(\psi(\xi, s)) \geq r^{1 / \alpha}(\xi) x^{(n-1)}(\xi) \mu_{l, n-2}(\xi, \psi(\xi, s))
$$

and so

$$
f(x(\psi(\xi, s))) \geq \operatorname{er}(\xi)\left(x^{(n-1)}(\xi)\right)^{\alpha} \mu_{l, n-2}^{\alpha}(\xi, \psi(\xi, s)) .
$$

Combining Equations (1) and (17), we obtain

$$
\left(r(\xi)\left(x^{(n-1)}(\xi)\right)^{\alpha}\right)^{\prime}+\varrho \int_{a}^{b} \rho(\xi, s) r(\xi)\left(x^{(n-1)}(\xi)\right)^{\alpha} \mu_{l, n-2}^{\alpha}(\xi, \psi(\xi, s)) \mathrm{d} s \leq 0
$$

or equivalently,

$$
\left(r(\xi)\left(x^{(n-1)}(\xi)\right)^{\alpha}\right)^{\prime}+\varrho r(\xi)\left(x^{(n-1)}(\xi)\right)^{\alpha} \int_{a}^{b} \rho(\xi, s) \mu_{l, n-2}^{\alpha}(\xi, \psi(\xi, s)) \mathrm{d} s \leq 0 .
$$

If we set $\Phi:=r(\xi)\left(x^{(n-1)}(\xi)\right)^{\alpha}$, then (19) reduces to

$$
\Phi^{\prime}(\xi)+\varrho \Phi(\xi) \int_{a}^{b} \rho(\xi, s) \mu_{l, n-2}^{\alpha}(\xi, \psi(\xi, s)) \mathrm{d} s \leq 0 .
$$

Using Grönwall inequality, we obtain

$$
\Phi(v) \geq \Phi(v) \exp \left(\int_{v}^{v}\left(\varrho \int_{a}^{b} \rho(u, s) \mu_{l, n-2}^{\alpha}(u, \psi(u, s)) \mathrm{d} s\right) \mathrm{d} u\right) .
$$

Therefore,

$$
\begin{aligned}
x^{(n-1)}(v) \geq & \left(\frac{1}{r(v)} \exp \left(\int_{v}^{v}\left(\varrho \int_{a}^{b} \rho(u, s) \mu_{l, n-2}^{\alpha}(u, \psi(u, s)) \mathrm{d} s\right) \mathrm{d} u\right)\right)^{1 / \alpha} \\
& \times\left(r^{1 / \alpha}(v) x^{(n-1)}(v)\right) .
\end{aligned}
$$

Integrating this inequality from $u$ to $v$ and by using (15), we have

$$
\begin{aligned}
-x^{(n-2)}(u) \geq & \int_{u}^{v}\left(\frac{1}{r(v)} \exp \left(\int_{v}^{v}\left(\varrho \int_{a}^{b} \rho(u, s) \mu_{l, n-2}^{\alpha}(u, \psi(u, s)) \mathrm{d} s\right) \mathrm{d} u\right)\right)^{1 / \alpha} \mathrm{d} v \\
& \times\left(r^{1 / \alpha}(v) x^{(n-1)}(v)\right) \\
\geq & r^{1 / \alpha}(v) x^{(n-1)}(v) \mu_{l+1,0}(v, u) .
\end{aligned}
$$

Integrating the previous inequality $(n-2)$ times from $u$ to $v$, we conclude that

$$
x(u) \geq r^{1 / \alpha}(v) x^{(n-1)}(v) \mu_{l+1, n-2}(v, u) .
$$

Thus, the proof is complete. 
Theorem 2. Assume that $\mu_{l, k}$ is defined as in Lemma 4. If, for some $l \in \mathbb{N}$,

$$
\limsup _{\xi \rightarrow \infty} \int_{\psi(\xi, b)}^{\xi} \int_{a}^{b} \rho(u, s) \mu_{l, n-2}^{\alpha}(\psi(\xi, s), \psi(u, s)) \mathrm{d} s \mathrm{~d} u>1,
$$

then $X_{D}^{+}$is empty.

Proof. Assume the contrary that $x \in X_{D}^{+}$. Then, there exists a $\xi_{1} \geq \xi_{0}$ such that $x(\xi)>0$ and $x(\psi(\xi, s))>0$ for all $\xi \geq \xi$. It follows from Lemma 4 that (14) holds. Integrating Equation (1) from $\psi(\xi, b)$ to $\xi$, we obtain

$$
r(\xi)\left(x^{(n-1)}(\xi)\right)^{\alpha}-r(\psi(\xi, b))\left(x^{(n-1)}(\psi(\xi, b))\right)^{\alpha}=-\int_{\psi(\xi, b)}^{\xi} \int_{a}^{b} \rho(u, s) f(x(\psi(u, s))) \mathrm{d} s \mathrm{~d} u .
$$

From the definition of $f$, we find

$$
r(\psi(\xi, b))\left(x^{(n-1)}(\psi(\xi, b))\right)^{\alpha} \geq \int_{\psi(\xi, b)}^{\xi} \varrho \int_{a}^{b} \rho(u, s) x^{\alpha}(\psi(u, s)) \mathrm{d} s \mathrm{~d} u .
$$

Using (14) with $u=\psi(u, s)$ and $v=\psi(\xi, s)$, we get that

$$
x(\psi(u, s)) \geq r^{1 / \alpha}(\psi(\xi, s)) x^{(n-1)}(\psi(\xi, s)) \mu_{l, n-2}(\psi(\xi, s), \psi(u, s)),
$$

which with (22), gives

$$
\varrho \int_{\psi(\xi, b)}^{\xi} \int_{a}^{b} \rho(u, s) \mu_{l, n-2}^{\alpha}(\psi(\xi, s), \psi(u, s)) \mathrm{d} s \mathrm{~d} u \leq 1,
$$

a contradiction. Thus, the proof is complete.

Theorem 3. Assume that $\mu_{l, k}$ is defined as in Lemma 4. If there exists a function $\Psi \in C\left(\left[\xi_{0}, \infty\right),(0, \infty)\right)$ such that $\Psi(\xi)<\xi, \psi(\xi, s)<\Psi(\xi)$ and the delay differential equation

$$
\omega^{\prime}(\xi)+\varrho\left(\int_{a}^{b} \rho(\xi, s) \mu_{l, n-2}^{\alpha}(\Psi(\xi), \psi(\xi, s)) \mathrm{d} s\right) \omega(\Psi(\xi))=0
$$

is oscillatory for some $l \in \mathbb{N}$, then $X_{D}^{+}$is empty.

Proof. Assume the contrary that $x \in X_{D}^{+}$. Then, there exists a $\xi_{1} \geq \xi_{0}$ such that $x(\xi)>0$ and $x(\psi(\xi, s))>0$ for all $\xi \geq \xi_{1}$. It follows from Lemma 4 that (14) holds. Using (14) with $u=\psi(\xi, s)$ and $v=\Psi(\xi)$, we get that

$$
x(\psi(\xi, s)) \geq r^{1 / \alpha}(\Psi(\xi)) x^{(n-1)}(\Psi(\xi)) \mu_{l, n-2}(\Psi(\xi), \psi(\xi, s)) .
$$

Thus, from Equation (1), we obtain

$$
\left(r(\xi)\left(x^{(n-1)}(\xi)\right)^{\alpha}\right)^{\prime}+\varrho r(\Psi(\xi))\left(x^{(n-1)}(\Psi(\xi))\right)^{\alpha} \int_{a}^{b} \rho(\xi, s) \mu_{l, n-2}^{\alpha}(\Psi(\xi), \psi(\xi, s)) \mathrm{d} s \leq 0 .
$$

If we set $\omega:=r\left(x^{(n-1)}\right)^{\alpha}$, then (24) becomes

$$
\omega^{\prime}(\xi)+\varrho\left(\int_{a}^{b} \rho(\xi, s) \mu_{l, n-2}^{\alpha}(\Psi(\xi), \psi(\xi, s)) \mathrm{d} s\right) \omega(\Psi(\xi)) \leq 0 .
$$


Thus, the last inequality has a positive solution. Using Theorem 1 in [10], we see that (23) also has a positive solution, which is a contradiction. The proof is complete.

Corollary 2. Assume that $\mu_{l, k}$ is defined as in Lemma 4. If there exists a function $\Psi \in C\left(\left[\xi_{0}, \infty\right),(0, \infty)\right)$ such that $\Psi(\xi)<\xi, \psi(\xi, s)<\Psi(\xi)$ and

$$
\liminf _{\xi \rightarrow \infty} \int_{\Psi(\xi)}^{\xi} \varrho \int_{a}^{b} \rho(u, s) \mu_{l, n-2}^{\alpha}(\Psi(u), \psi(u, s)) \mathrm{d} s \mathrm{~d} u>\frac{1}{\mathrm{e}} .
$$

Then $X_{D}^{+}$is empty.

Proof. Applying a well-known criterion Theorem 2 in [13] for first-order delay differential Equation (23) to be oscillatory, we obtain immediately the criterion (25).

Example 2. Consider the third-order differential Equation (12). Then, we see that

$$
\begin{gathered}
\mu_{0,0}(v, u)=v-u, \mu_{0,1}(v, u)=\frac{1}{2}(v-u)^{2}, \\
\mu_{1,0}(v, u)=\varrho \rho_{0} \frac{(1-\epsilon)^{3}}{2} v \ln \frac{v}{u}
\end{gathered}
$$

and

$$
\mu_{1,1}(v, u)=\varrho \rho_{0} \frac{(1-\epsilon)^{3}}{2} v\left(v-u\left(1+\ln \frac{v}{u}\right)\right) .
$$

Thus, by choosing $k=0, l=1$ and $\Psi(\xi):=\frac{3}{2} \epsilon \xi$, condition (25) reduces to

$$
3 \varrho^{2} \rho_{0}^{2} \epsilon^{2}(1-\epsilon)^{4}\left(\frac{1}{2}-\ln \frac{3}{2}\right) \ln \frac{2}{3 \epsilon}>\frac{4}{\mathrm{e}} .
$$

From Corollary 2, we see that $X_{D}^{+}$is empty if condition (26) satisfies.

Theorem 4. Assume that $\eta_{k}$ and $\mu_{l, k}$ are defined as in Lemmas 3 and 4 , respectively. If there exists a function $\Psi \in C\left(\left[\xi_{0}, \infty\right),(0, \infty)\right)$ such that $\Psi(\xi)<\xi$ and $\psi(\xi, s)<\Psi(\xi)$, for some $\delta_{k} \in(0,1)$ and some $k, l \in \mathbb{N}$ and the delay differential Equations (9) and (23) are oscillatory, then, every solution of Equation (1) is oscillatory.

Corollary 3. Assume that $\eta_{k}$ and $\mu_{l, k}$ are defined as in Lemmas 3 and 4 , respectively. Then, every solution of Equation (1) is oscillatory if one of the following conditions is provided:

(a) Equations (11) and (21)

(b) Equations (11) and (25)

for some $\delta_{k} \in(0,1)$ and some $k, l \in \mathbb{N}$.

Remark 2. From the results in Examples 1 and 2, we note that the conditions (11) and (25) reduce to (13) and (26). Therefore, using Corollary 3-(b), we conclude that every solution of (12) is oscillatory if (13) and (26) hold.

\section{Conclusions}

Through applying an iterative approach in this work, we established sharper estimates for increasing/decreasing positive solutions of Equation (1). Using the principles of comparison, we obtained new oscillation criteria that can be used to test for oscillations, even when the previously known criteria fail to apply.

The memory effect may also appear non-locally when certain fractional derivatives with power, exponential and Mittag-Leffler laws are possibly applied. So, it would be interesting to extend the results of this paper to the fractional delay differential equations, see [28-32]. 
Author Contributions: Formal analysis: A.M. and T.A.; investigation: A.M. and O.M.; supervision: O.M. and E.M.E.; writting-original draft: A.M. and T.A.: writting-review\&editting: T.A., O.M. and E.M.E. The authors claim to have contributed equally and significantly in this paper. All authors have read and agreed to the published version of the manuscript.

Funding: This research received no external funding.

Acknowledgments: The authors thank the reviewers for their useful comments, which led to the improvement of the content of the paper.

Conflicts of Interest: The authors declare no conflict of interest.

\section{References}

1. Hale, J.K. Theory of Functional Differential Equations; Springer: New York, NY, USA, 1977.

2. Cairone, F.; Gagliano, S.; Bucolo, M. Experimental study on the slug flow in a serpentine microchannel. Exp. Therm. Fluid Sci. 2016, 76, 34-44. [CrossRef]

3. Agarwal, R.P.; Grace, S.R.; O'Regan, D. Oscillation Theory for Difference and Functional Differential Equations; Springer Science \& Business Media: Berlin/Heidelberg, Germany, 2000.

4. Agarwal, R.P.; Shieh, S.L.; Yeh, C.C. Oscillation criteria for second order retarded differential equations. Math. Comput. Model. 1997, 26,1-11. [CrossRef]

5. Li, T.; Rogovchenko, Y.V.; Zhang, C. Oscillation of fourth-order quasilinear differential equations. Math. Bohem. 2015, 140, 405-418. [CrossRef]

6. Li, T.; Zhang, C.; Thandapani, E. Asymptotic behavior of fourth-order neutral dynamic equations with noncanonical operators. Taiwanese J. Math. 2014, 18, 1003-1019. [CrossRef]

7. Liu, Q.; Bohner, M.; Grace, S.R.; Li, T. Asymptotic behavior of even-order damped differential equations with p-Laplacian like operators and deviating arguments. J. Inequal. Appl. 2016, 2016, 321. [CrossRef]

8. Moaaz, O.; Elabbasy, E.M.; Muhib, A. Oscillation criteria for even-order neutral differential equations with distributed deviating arguments. Adv. Differ. Equ. 2019, 2019, 297. [CrossRef]

9. Moaaz, O.; Muhib, A. New oscillation criteria for nonlinear delay differential equations of fourth-order. Appl. Math. Comput. 2020, 377, 125192. [CrossRef]

10. Philos, C. On the existence of nonoscillatory solutions tending to zero at $\infty$ for differential equations with positive delays. Arch. Math. (Basel) 1981, 36, 168-178. [CrossRef]

11. Moaaz, O.; Park, C.; Muhib, A.; Bazighifan, O. Oscillation criteria for a class of even-order neutral delay differential equations. J. Appl. Math. Comput. 2020, 63, 607-617. [CrossRef]

12. Moaaz, O.; Muhib, S.F.A. New comparison theorems for the nth order neutral differential equations with delay inequalities. Mathematics 2020, 8, 454. [CrossRef]

13. Kitamura, Y.; Kusano, T. Oscillation of first-order nonlinear differential equations with deviating arguments. Proc. Am. Math. Soc. 1980, 78, 64-68. [CrossRef]

14. McKean, H.P. Nagumo's equation. Adv. Math. 1970, 4, 209-223. [CrossRef]

15. Bohner, M.; Grace, S.R.; Sager, I.; Tunc, E. Oscillation of third-order nonlinear damped delay differential equations. Appl. Math. Comput. 2016, 278, 21-32. [CrossRef]

16. Padhi, S.; Pati, S. Theory of Third-Order Differential Equations; Springer: New Delhi, India, 2014.

17. Chatzarakis, G.E.; Grace, S.R.; Jadlovska, I. Oscillation criteria for third-order delay differential equations. Adv. Differ. Equ. 2017, 2017, 330. [CrossRef]

18. Baculikova, B.; Dzurina, J. On the oscillation of odd order advanced differential equations. Bound. Value Probl. 2014, 2014, 214. [CrossRef]

19. Dzurina, J.; Grace, S.R.; Jadlovska, I. On nonexistence of kneser solutions of third-order neutral delay differential equations. Appl. Math. Lett. 2019, 88, 193-200. [CrossRef]

20. Moaaz, O.; Baleanu, D.; Muhib, A. New aspects for non-existence of kneser solutions of neutral differential equations with odd-order. Mathematics 2020, 8, 494. [CrossRef]

21. Agarwal, R.P.; Bohner, M.; Li, T.; Zhang, C. Oscillation of third-order nonlinear delay differential equations. Taiwan. J. Math. 2013, 17, 545-558. [CrossRef]

22. Baculikova, B.; Dzurina, J. Oscillation of third-order nonlinear differential equations. Appl. Math. Lett. 2011, 24, 466-470. [CrossRef] 
23. Baculikova, B.; Dzurina, J. Oscillation of third-order functional differential equations. Electron. J. Qual. Theory Differ. Equ. 2010, 2010, 43. [CrossRef]

24. Moaaz, O.; Qaraad, B.; El-Nabulsi, R.A.; Bazighifan, O. New results for kneser solutions of third-order nonlinear neutral differential equations. Mathematics 2020, 8, 686. [CrossRef]

25. Moaaz, O.; Awrejcewicz, J.; Muhib, A. Establishing new criteria for oscillation of odd-order nonlinear differential equations. Mathematics 2020, 8, 937. [CrossRef]

26. Vidhyaa, K.S.; Graef, J.R.; Thandapani, E. New oscillation results for third-order half-linear neutral differential equations. Mathematics 2020, 8, 325. [CrossRef]

27. Li, T.; Rogovchenko, Y.V. Asymptotic behavior of an odd-order delay differential equation. Bound. Value Probl. 2014, 2014, 107. [CrossRef]

28. Grace, S.R.; Agarwal, R.P.; Wong, P.J.Y.; Zafer, A. On the oscillation of fractional differential equations. Fract. Calc. Appl. Anal. 2012, 15, 222-231. [CrossRef]

29. Abdalla, B.; Abdeljawad, T. On the oscillation of Hadamard fractional differential equations. Adv. Differ. Equ. 2018, 2018, 409. [CrossRef]

30. Abdalla, B. Oscillation of differential equations in the frame of nonlocal fractional derivatives generated by conformable derivatives. Adv. Differ. Equ. 2018, 2018, 107. [CrossRef]

31. Chen, D.X. Oscillation criteria of fractional differential equations. Adv. Differ. Equ. 2012, 2012, 33. [CrossRef]

32. Abdalla, B.; Abdeljawad, T. On the oscillation of Caputo fractional differential equations with Mittag-Leffler nonsingular kernel. Chaos Solitons Fractals 2019, 127, 173-177. [CrossRef]

Sample Availability: Samples of the compounds ...... are available from the authors.

(C) 2020 by the authors. Licensee MDPI, Basel, Switzerland. This article is an open access article distributed under the terms and conditions of the Creative Commons Attribution (CC BY) license (http:/ / creativecommons.org/licenses/by/4.0/). 